\title{
Detergent-resistant plasma membrane proteome to elucidate microdomain functions in plant cells
}

\author{
Daisuke Takahashi ${ }^{1}$, Yukio Kawamura, ${ }^{1,2}$ and Matsuo Uemura ${ }^{1,2 *}$ \\ 1 United Graduate School of Agricultural Sciences, Iwate University, Morioka, Japan \\ ${ }^{2}$ Cryobiofrontier Research Center, Faculty of Agriculture, I wate University, Morioka, Japan
}

\section{Edited by:}

Nicolas L. Taylor, The University of Western Australia, Australia

\section{Reviewed by:}

Stefanie Wienkoop, University of Vienna, Austria

Natalia V. Bykova, Memorial University of Newfoundland, Canada

\section{*Correspondence:}

Matsuo Uemura, Cryobiofrontier Research Center, Faculty of Agriculture, Iwate University, 3-18-8 Ueda, Morioka, Iwate 020-8550, Japan

e-mail: vemura@iwate-u.ac.jp
Although proteins and lipids have been assumed to be distributed homogeneously in the plasma membrane (PM), recent studies suggest that the PM is in fact non-uniform structure that includes a number of lateral domains enriched in specific components (i.e., sterols, sphingolipids, and some kind of proteins). These domains are called as microdomains and considered to be the platform of biochemical reaction center for various physiological processes. Microdomain is able to be extracted as detergent-resistant membrane (DRM) fractions, and DRM fractions isolated from some plant species have been used for proteome and other biochemical characterizations to understand microdomain functions. Profiling of sterol-dependent proteins using a putative microdomain-disrupting agent suggests specific lipid-protein interactions in the microdomain. Furthermore, DRM proteomes dynamically respond to biotic and abiotic stresses in some plant species. Taken together, these results suggest that DRM proteomic studies provide us important information to understand physiological functions of microdomains that are critical to prosecute plant's life cycle successfully in the aspect of development and stress responses.

Keywords: detergent-resistant plasma membrane (DRM), microdomain, lipid raft, proteome, biotic stress, abiotic stress

\section{INTRODUCTION}

The plasma membrane (PM) is a typical cellular membrane with selective permeability and surrounds all organelles and cellular substances. Therefore, the PM is thought to be the most important cellular membrane due to relationships to various important cellular processes including cell division, differentiation, and biotic/abiotic stress adaptation. The PM contains a variety of proteins associated with transport, signaling, cytoskeleton construction, metabolism, and stress protection in the form of transmembrane, peripheral, and lipid modified types.

Lateral distribution of these membrane proteins has been described by diffusion of each lipid and protein molecule which is proposed as fluid mosaic model (Singer and Nicolson, 1972). In addition to this hypothesis, Simons and Ikonen (1997) proposed functional microdomain of the PM. In PM microdomain hypothesis, it is considered that microdomain is organized with highly hydrophobic lipids such as sterols and sphingolipids, and specific proteins with defined functions (Brown and London, 1998, 2000; London and Brown, 2000). In animal cells, one of microdomain function is considered to be a scaffold in association with signaling complex, membrane trafficking, and transport (Simons and Ikonen, 1997; Simons and Toomre, 2000; Lingwood and Simons, 2010). Experimentally, microdomain can be obtained as nonionic detergent-resistant membrane (DRM) fraction due to their own hydrophobic properties (Schroeder et al., 1994; Simons and Ikonen, 1997; Brown and London, 1998).

Peskan et al. (2000) reported for the first time isolation of DRM fractions from plant materials using tobacco leaves. After this report, the isolation of DRM fractions have been reported with other plant species such as tobacco, Arabidopsis thaliana, leek, Medicago truncatula, Solanum tuberosum, rice, oat, and rye (Mongrand etal., 2004; Borner et al., 2005; Morel et al., 2006; Laloi et al., 2007; Lefebvre et al., 2007; Krügel et al., 2008; Fujiwara et al., 2009; Minami et al., 2009; Takahashi et al., 2012). Some physiological studies showed possibilities that microdomain is involved in pollen tube tip growth, intracellular virus movement, and clathrin-independent endocytotic pathway (Liu et al., 2009; Raffaele et al., 2009; Li et al., 2012). In addition to these functions, PM microdomain may have roles in cell wall polysaccharide synthesis in hybrid aspen (Bessueille et al., 2009).

There have been attempts to identify microdomain-associated proteins for elucidation of novel microdomain-dependent regulatory mechanisms on cellular physiological processes in plant. Most of these studies were 2D or 1D electrophoresis gel-based proteomics or nano-LC-MS/MS-based shotgun proteomics using microdomain-enriched DRM fraction. In addition to DRM fraction, methyl- $\beta$-cyclodextrin $(\mathrm{m} \beta \mathrm{CD})$, which is known as a sterol chelator and, hence, a sterol-dependent microdomain disrupter, was used to characterize how protein was associated with the primary microdomain lipid, sterol (Kierszniowska et al., 2009). Comprehensive analyses of DRM proteomes may contribute to demonstrate the importance of lateral segregation of proteins in plant PM microdomains. Ultimately, these results may lead to new findings of plant cellular homeostasis system such as signaling machinery, transport regulation, and novel response system against perception of biotic stress such as fungal infection and abiotic stress such as drought, salt, light, nutrition, and temperature. 


\section{DETERGENT-RESISTANT MEMBRANE FRACTION AS A BIOCHEMICAL SAMPLE FOR OBTAINING INFORMATION ASSOCIATED WITH PLASMA MEMBRANE MICRODOMAIN}

To analyze biochemical properties, the extraction of DRM fractions from the PM is considered to be the only way to prepare microdomain samples (Figure 1). DRM fractions were isolated from a number of plant species and tissues as described above, and the preparation protocols of DRM fractions are in general quite similar regardless of plant species. First, a highly pure PM fraction is prepared using a two-phase partition system and then treated with $1 \%(\mathrm{w} / \mathrm{v})$ Triton X-100 detergent at low temperature (on ice or $4^{\circ} \mathrm{C}$ ) for $30 \mathrm{~min}$. Next, treated membrane fraction is subjected to sucrose density gradient centrifugation. After centrifugation, white band appeared at the interface of sucrose layers recovered and collected by centrifugation. Precipitated membrane fraction is suspended in a proper buffer as DRM fraction. Because unknown artificial effects might be caused

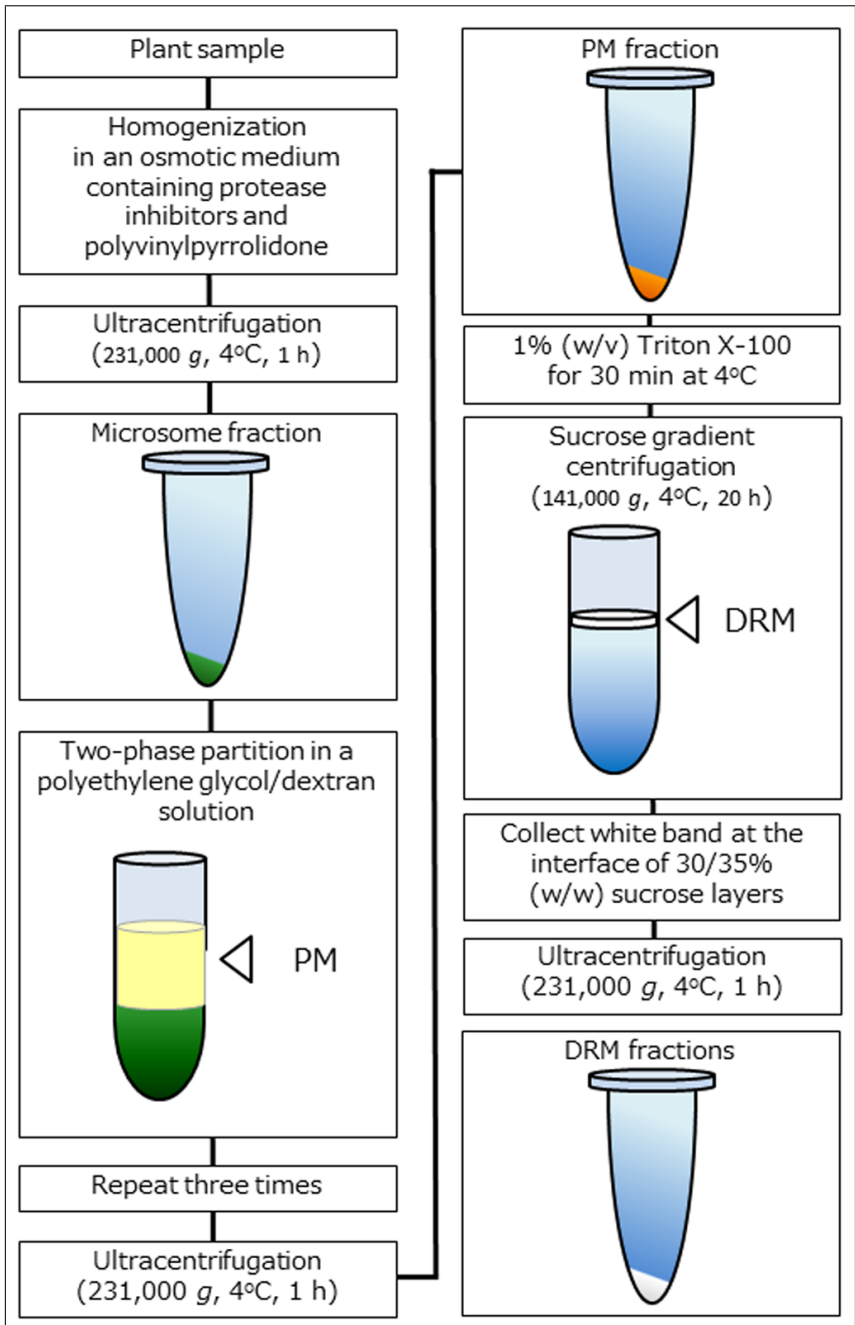

FIGURE 1 | Schematic representation of DRM extraction in plants. Overview of the DRM extraction procedure. DRM fractions are obtained from purified PM fractions by the $1 \%(\mathrm{w} / \mathrm{v})$ Triton X-100 treatment and subsequent sucrose density gradient centrifugation. due to detergent treatment at low temperature, some researchers concerned that intact microdomains that function in vivo are not extracted by the widely adapted preparation protocols (Tanner etal., 2011). Nevertheless, DRM fraction is a useful tool for estimating microdomain functions associated with specific components. Many microdomain-related phenomena have been elucidated in DRM and non-DRM fractions, and experiments with DRM fraction is apparently one of the most effective ways to determine specific functions in relation to microdomains in PM (Lingwood and Simons, 2007).

Proteomics approaches of DRM proteins is well-conducted in various organisms and, further, quantification of DRM and non-DRM proteins is also reported in some plant species using isotope labeling, 2D difference gel electrophoresis (2D-DIGE), and label-free quantification software (Kierszniowska et al., 2009; Minami et al., 2009; Takahashi et al., 2012). However, there are still difficulties in quantitative determination of a large number of proteins correctively using proteomic approached. This is in part because solubilization of membrane proteins including those localized in the PM as well as DRM may not be consistent in a series of experiments due to hydrophobic characteristics of the proteins and assignment of peptide fragments to the appropriate protein may not be accurate in some species for which we have not yet completed genome sequencing. It is necessary to combine another approaches (such as immunochemical and biochemical approaches) to obtain the amount of proteins in the membrane accurately.

\section{FUNCTION OF THE PM MICRODOMAIN}

Detergent-resistant membrane proteomes have been determined in some plant species (Mongrand et al., 2004; Shahollari et al., 2004; Borner et al., 2005; Morel et al., 2006; Lefebvre et al., 2007; Fujiwara et al., 2009; Minami et al., 2009; Stanislas et al., 2009; Takahashi et al., 2012). Comparisons of DRM proteomes from these plant species indicated that DRM protein functions are very similar among plant species: DRM fractions contain many transporters, proteins associated with membrane vesicle trafficking processes and cytoskeleton such as $\mathrm{H}^{+}$-ATPases, aquaporins, clathrins, actins, and tubulins. Further, microscopic observations and biochemical analyses of DRM fractions or intact plant cells implied that microdomains play some functional roles in the physiological aspects. Table 1 summarizes proteins that were found in common in some plant species on papers published so far. Localization or function of some of these proteins in distinct regions in the PM was further confirmed by additional approaches either morphologically or biochemically.

As an example of functional involvement in developmental process, Liu et al. (2009) reported the involvement of microdomain in pollen tube tip growth. Using sterol-enriched microdomains in pollen tube using one of microdomain-staining lipophilic styryl dyes, di-4-ANEPPDHQ, they clearly revealed localization of NADPH oxidase in microdomain. From the results, they suggested that one of predicted microdomain properties (i.e., clustering of specific, hydrophobic lipids and proteins) is required for NADPH oxidase activity and polarization of sterol-enriched microdomain regulates NADPH oxidase-dependent reactive oxygen species signaling. Ultimately, polar growth of pollen tube tip 


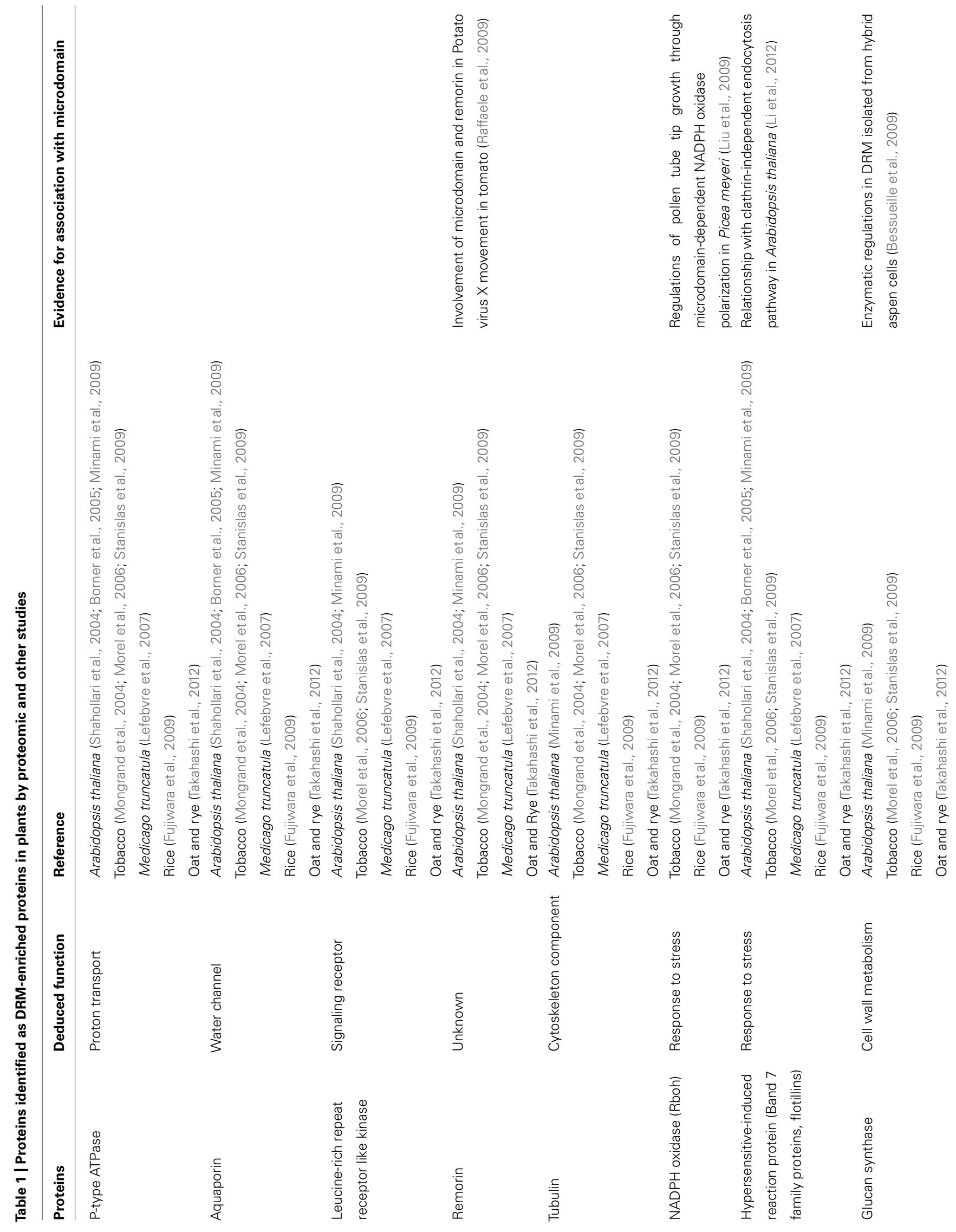


may be modulated by the localization of proteins in microdomain. In addition to plant pollen tip, polarization of microdomain in hyphal tip of Candida albicans was also observed (Martin and Konopka, 2004). These data together suggest that characteristics of microdomain are common in the function on cell polarization among various species not only plants but also microorganisms.

A recent study also suggested that microdomain is related to intracellular membrane trafficking. Arabidopsis Flot1 is a DRMassociated protein that was identified in DRM proteome (Borner et al., 2005). Li et al. (2012) observed that Flot1 showed patch-like localization on PM using electron microscopic technique. They further showed that Flot 1 is participated in endocytic vesicle formation but gold-conjugated antibody of Flot 1 does not co-localize with clathrin light chain. It means that Flot1 plays some roles in a microdomain-associated but clathrin-independent endocytosis pathway. Considering that RNA interference of Flot1 results in the defect of seedling development, microdomain-Flot1 mediated vesicle trafficking has important implications for seedling development such as root hair elongation regulated by vesicle trafficking (Ovecka et al., 2010).

According to protein clustering in microdomain, proteomic and subsequent enzymatic characterizations of DRM fraction from hybrid aspen cells strongly suggested the involvement of DRM in cell wall polysaccharide synthesis (Bessueille et al., 2009). DRM from hybrid aspen was enriched in glucan synthases such as callose and cellulose synthase, and, surprisingly, 73\% of total glucan synthase activities of PM were detected in DRM. They concluded that microdomain is functional platform for cell wall component synthesis and controls cell morphogenesis.

Detailed analysis of M. truncatula DRM showed considerable differences in DRM fraction and the total PM fraction (Lefebvre et al., 2007). This study showed that free sterols, sphingolipids, and steryl glycosides are highly enriched in DRM fractions. These results are consistent with previous studies with tobacco and $A$. thaliana (Mongrand et al., 2004; Borner et al., 2005). In addition to lipids, global survey of DRM proteins were performed and revealed that signaling-, transport-, redox-, cytoskeleton-, trafficking-, and cell wall-related proteins were enriched in DRM, most of which were also found in early works of plant DRM protein identification (Mongrand et al., 2004; Shahollari et al., 2004; Borner et al., 2005; Morel et al., 2006). Proteome profiling of M. truncatula DRM further indicated the possible presence of microdomaindependent redox regulation system and microdomain platform for signaling.

As described above, sterols are one of the primary components of microdomain-enriched DRM fractions in both animal and plant cells. Kierszniowska et al. (2009) applied $\mathrm{m} \beta \mathrm{CD}$ to isolated Arabidopsis DRM fractions to analyze sterol-dependent enrichment of DRM proteins. $\mathrm{m} \beta \mathrm{CD}$ is a sterol-removing cyclic oligosaccharide and $\mathrm{m} \beta \mathrm{CD}$ treatment disrupts the organization of membrane microdomain. Proteomic analysis of the $\mathrm{m} \beta \mathrm{CD}$ treated and untreated DRM fractions revealed that cell wall-related and glycosylphosphatidylinositol anchored proteins (a class of lipid-modified proteins) were changed by sterol depletion. Thus, these results strongly suggest that sterol is an important factor for segregation of specific proteins into DRM fraction and
PM is "phase-separated" to form specific domains (i.e., sterolenriched microdomains, Xu et al., 2001). As shown in these studies, proteome analysis has been used for estimating microdomain functions in plant cells for the past decades and, therefore, greatly contributed to elucidation of microdomain-associated physiological functions in plant cells.

\section{DRM PROTEOME ON BIOTIC STRESS RESPONSE}

Plant proteomic studies for elucidating microdomain function have been carried out intensively in the research area of plantpathogen interactions. The possibility of lipid microdomainpathogen interactions was first reported by Bhat etal. (2005). The authors suggest that fungal pathogen (Blumeria graminis f.sp. hordei) recognizes barley mildew resistance locus o (Mlo) that seems to re-localize as microdomain-like structure at pathogen invasion site. In addition to pathogen infection, plant immune responses against biotic stress may be supported by functional microdomain. Fujiwara et al. (2009) successfully identified 192 proteins from DRM proteome analysis in rice suspension cultured cells that were pre-transformed with constitutively active OsRac. OsRacl is one of the Rac/Rop GTPase family proteins and regulates rice immunity as a key regulator (Kawasaki et al., 1999; Ono et al., 2001; Wong et al., 2004; Lieberherr et al., 2005; Kim et al., 2012). Shift of OsRacl to DRM fractions was found after elicitor treatment. At the same time, DRM proteome suggests that microdomain exists as platform for rice innate immunity. Actually, receptor-like kinases $(R L K)$, disease resistance proteins and band7 family proteins, members of disease-related proteins, were detected in rice DRM fractions as well as some other plant species (Borner et al., 2005; Morel et al., 2006; Fujiwara et al., 2009; Minami et al., 2009). Interactions between OsRac1 and those proteins may occur during initial immunity process against biotic stimuli.

Mongrand et al. (2004) also suggest from proteomics of DRM that microdomain isolated as DRM fractions has important functions in plant defense responses because tobacco DRM proteome contains a variety of defense-related proteins such as remorin, NtrbohD, and Ntrac5. Some physiological studies further indicated that DRM-enriched proteins are associated with plant-pathogen interactions. Remorin is the most characterized and a representative DRM protein and Raffaele et al. (2009) reported interesting results that remorin is associated with intercellular virus movement. Solanaceae remorin was fractionated into DRM fraction, which is also reported in tobacco DRM proteomics (Mongrand et al., 2004; Morel et al., 2006; Stanislas et al., 2009) as well as oat and rye proteomics (Takahashi et al., 2012). Interestingly, the distribution of remorin on the PM was represented as patch-like patterns and disappeared when $\mathrm{m} \beta \mathrm{CD}$ was added to the sample. These results strongly suggest that DRM fractions partly reflect intact microdomain. Raffaele et al. (2009) also showed that remorin is localized in plasmodesmata and its accumulation levels affect cell-to-cell transfer of Potato virus X (PVX) through plasmodesmata. Detailed analysis of DRM proteome against elicitor signaling in tobacco BY-2 cells revealed that the DRM enrichment of cell trafficking related proteins (dynamins) and a signaling protein (14-3-3 protein) altered after cryptogein treatment (Stanislas et al., 2009). These studies clearly indicate that DRM proteomics has potential to find new factors of elicitor signaling pathway and 
their functions in plants, and DRM proteome has methodological significance in approach for findings of novel microdomain functions on plant pathology.

\section{DRM PROTEOME ON ABIOTIC STRESS RESPONSE}

Abiotic stress response and adaptation mechanism in association with microdomain is not well characterized. The only study showing changes of DRM compositions in response to abiotic stimuli was with Arabidopsis leaves reported by Minami et al. (2009). They performed Arabidopsis DRM proteomic analysis to find the possibilities of microdomain functions for adaptation to freezing temperature. Plants can increase survival at severe freezing temperatures by sensing non-freezing low temperature and subsequently reconstituting cellular processes (called as cold acclimation; Guy, 1990; Sharma et al., 2005). Although there are a number of papers revealing considerable changes of PM compositions during cold acclimation (Uemura and Yoshida, 1984; Lynch and Steponkus, 1987; Webb etal., 1994; Kawamura and Uemura, 2003; Uemura et al., 2006), analysis of DRM compositions during cold acclimation was conducted in very few studies. Using a combination of 1D sodium dodecyl sulfate polyacrylamide gel electrophoresis (SDS-PAGE), 2D-DIGE and Liquid chromatography-tandem mass spectrometry (LCMS/MS) and Matrix-assisted laser desorption/ionisation-time of flight mass spectrometry (MALDI-TOF/MS) analysis, Minami et al. (2009) demonstrated that proteomic profiles of DRM fractions altered significantly during cold acclimation. The cold acclimation-responsive proteins include synaptotagmin protein homolog, tubulin and P-type ATPase. Each protein is considered to have important roles in cold acclimation process from previous studies. For example, synaptotagmin homolog SYT1 was identified in DRM and increased after cold acclimation. SYT1 is related to calcium-dependent PM resealing (or repairing) process when PM destruction occurs due to freeze-induced mechanical stress imposed by extracellular ice formation (Yamazaki et al., 2008). In addition to SYT1, other membrane fusion-related proteins such as syntaxin were identified in oat and rye DRM (Takahashi et al., 2012). Disassembly of microtubule consisting of tubulins is suggested to be important for inducing cold acclimation process (Abdrakhamanova etal., 2003). Enhancement of ATPase activity in PM during cold acclimation is one of the well-known reactions in some plant species (Ishikawa and Yoshida, 1985; Martz et al., 2006).

How interactions between these proteins and microdomain properties affect cold acclimation processes, however, is still to be elucidated. We need to conduct additional physiological and microscopic experiments to understand responsiveness of microdomain and/or DRM proteins to cold acclimation. We have

\section{REFERENCES}

Abdrakhamanova, A., Wang, Q. Y., Khokhlova, L., and Nick, P. (2003). Is microtubule disassembly a trigger for cold acclimation? Plant Cell Physiol. 44, 676-686.

Bessueille, L., Sindt, N., Guichardant, M., Djerbi, S., Teeri, T. T., and Bulone, V. (2009). Plasma membrane

evidence from proteomic studies that there are several interesting abiotic stress-related proteins in DRM fractions such as RLKs, aquaporins, heat shock proteins, actins, and clathrins in various plants (Mongrand etal., 2004; Borner et al., 2005; Morel et al., 2006; Takahashi et al., 2012). To elucidate their contribution to abiotic stress sensing, signaling, and response, comprehensive proteomic analyses such as protein-protein interactions and posttranslational modifications of the proteins would be necessary and expected.

\section{FUTURE PERSPECTIVE}

Proteomic analyses of DRM fractions have been conducted and provided information for suggestive but important functions of PM microdomain in plants. Several physiological studies using both intact cells and isolated membrane fractions supported implications derived from proteomic analyses with DRM and added further interesting information on the roles of membrane microdomains. However, evidence of functional roles of microdomains in the PM is in a large part lacking. Now we are entering in next phase for elucidating microdomain characteristics and functions in plants. We need to consider morphology and dynamics of microdomains, physical and chemical state of PM proteins in microdomains from the perspective of post-translational modifications and molecular ultrastructure, and ultimately functional significance of microdomains in various events in plant's life. Development of microscopic and biochemical techniques, such as singlemolecule tracking and artificial membrane system, will help us to understand physiological roles of microdomain in plant cells.

Plants are immobile and, thus, perception and response to environmental stimuli are quite important for plant's life. The PM is thought to be the primary cellular compartment of these reactions because it surrounds intracellular organelles and the cytoplasm and transduces extracellular stimuli to the specific components in the cell. Microdomain is expected to play important roles in these processes. Thus, proteomic approaches will further provide useful information for understanding plant physiological responses and microdomain significance in the future.

\section{ACKNOWLEDGMENTS}

We thank Drs. Fukao and Masayuki Fujiwara (Nara Institute of Science and Technology) and Setsuko Komatsu (National Agriculture and Food Research Organization) for valuable discussions. This study was in part supported by Grants-in-Aids for Scientific Research from Ministry of Education, Culture, Sports, Science and Technology in Japan (\#22120003) and from Japanese Society for the Promotion of Sciences (\#24.7373 and \#24370018).

membrane microdomain. Proc. Natl. Acad. Sci. U.S.A. 102, 3135-3140.

Borner, G. H. H., Sherrier, D. J., Weimar, T., Michaelson, L. V., Hawkins, N. D., Macaskill, A., et al. (2005). Analysis of detergent-resistant membranes in Arabidopsis: evidence for plasma membrane lipid rafts. Plant Physiol. 137, 104-116.
Brown, D. A., and London, E. (1998). Function of lipid rafts in biological membranes. Annu. Rev. Cell Dev. Biol. 14, 111-136

Brown, D. A., and London, E. (2000). Structure and function of sphingolipid- and cholesterol-rich membrane rafts. J. Biol. Chem. 275, 17221-17224. 
Fujiwara, M., Hamada, S., Hiratsuka, M., Fukao, Y., Kawasaki, T., and Shimamoto, K. (2009). Proteome analysis of detergent-resistant membranes (DRMs) associated with OsRacl-mediated innate immunity in rice. Plant Cell Physiol. 50, 11911200.

Guy, C. (1990). Cold acclimation and freezing stress tolerance: role of protein metabolism. Annu. Rev. Plant Physiol. Plant Mol. Biol. 41, 187-223.

Ishikawa, M., and Yoshida, S. (1985). Seasonal changes in plasma membranes and mitochondria isolated from Jerusalem artichoke tubers: possible relationship to cold hardiness. Plant Cell Physiol. 26, 13311344.

Kawamura, Y., and Uemura, M. (2003). Mass spectrometric approach for identifying putative plasma membrane proteins of Arabidopsis leaves associated with cold acclimation. Plant J. 36, 141-154.

Kawasaki, T., Henmi, K., Ono, E., Hatakeyama, S., Iwano, M., Satoh, H., et al. (1999). The small GTP-binding protein rac is a regulator of cell death in plants. Proc. Natl. Acad. Sci. U.S.A. 96, 10922-10926.

Kierszniowska, S., Seiwert, B., and Schulze, W. X. (2009). Definition of Arabidopsis sterol-rich membrane microdomains by differential treatment with methylbeta-cyclodextrin and quantitative proteomics. Mol. Cell. Proteomics 8 , 612-623.

Kim, S.-H., Oikawa, T., Kyozuka, J., Wong, H. L., Umemura, K., KishiKaboshi, M., etal. (2012). The bHLH Rac immunityl (RAI1) is activated by OsRacl via OsMAPK3 and OsMAPK6 in rice immunity. Plant Cell Physiol. 53, 740-754.

Krügel, U., Veenhoff, L. M., Langbein, J., Wiederhold, E., Liesche, J., Friedrich, T., et al. (2008). Transport and sorting of the Solanum tuberosum sucrose transporter SUT1 is affected by posttranslational modification. Plant Cell 20, 2497-2513.

Laloi, M., Perret, A.-M., Chatre, L., Melser, S., Cantrel, C., Vaultier, M.N., et al. (2007). Insights into the role of specific lipids in the formation and delivery of lipid microdomains to the plasma membrane of plant cells. Plant Physiol. 143, 461-472.

Lefebvre, B., Furt, F., Hartmann, M.-A., Michaelson, L. V., Carde, J.-P., Sargueil-Boiron, F., et al. (2007). Characterization of lipid rafts from Medicago truncatula root plasma membranes: a proteomic study reveals the presence of a raftassociated redox system. Plant Physiol. 144, 402-418.

Li, R., Liu, P., Wan, Y., Chen, T., Wang, Q., Mettbach, U., et al. (2012). A membrane microdomain-associated protein, Arabidopsis Flot1, is involved in a clathrin-independent endocytic pathway and is required for seedling development. Plant Cell 24, 21052122.

Lieberherr, D., Thao, N. P., Nakashima, A., Umemura, K., and Kawasaki, T. (2005). A sphingolipid elicitorinducible mitogen-activated protein kinase is regulated by the small GTPase OsRacl and heterotrimeric G-protein in rice. Plant Physiol. 138, 1644-1652.

Lingwood, D., and Simons, K. (2007). Detergent resistance as a tool in membrane research. Nat. Protoc. 2, 2159 2165.

Lingwood, D., and Simons, K. (2010). Lipid rafts as a membraneorganizing principle. Science 327, 46-50.

Liu, P., Li, R.-L., Zhang, L., Wang, Q.-L., Niehaus, K., Baluska, F., et al. (2009). Lipid microdomain polarization is required for NADPH oxidasedependent ROS signaling in Picea meyeri pollen tube tip growth. Plant J. 60, 303-313.

London, E., and Brown, D. A. (2000). Insolubility of lipids in Triton X-100: physical origin and relationship to sphingolipid/cholesterol membrane domains (rafts). Biochim. Biophys. Acta 1508, 182-195.

Lynch, D. V., and Steponkus, P. L. (1987). Plasma membrane lipid alterations associated with cold acclimation of winter rye seedlings (Secale cereale L. cv Puma). Plant Physiol. 83, 761-767.

Martin, S. W., and Konopka, J. B. (2004). Lipid raft polarization contributes to hyphal growth in Candida albicans. Eukaryot. Cell 3, 675-684.

Martz, F., Sutinen, M.-L., Kiviniemi, S., and Palta, J. P. (2006). Changes in freezing tolerance, plasma membrane $\mathrm{H}^{+}$-ATPase activity and fatty acid composition in Pinus resinosa needles during cold acclimation and de-acclimation. Tree Physiol. 26 783-790.

Minami, A., Fujiwara, M., Furuto A., Fukao, Y., Yamashita, T. Kamo, M., etal. (2009). Alterations in detergent-resistant plasma membrane microdomains in Arabidopsis thaliana during cold acclimation. Plant Cell Physiol. 50, 341-359.

Mongrand, S., Morel, J., Laroche, J., Claverol, S., Carde, J.-P., Hartmann,
M.-A., etal. (2004). Lipid rafts in higher plant cells: purification and characterization of Triton $\mathrm{X}$-100-insoluble microdomains from tobacco plasma membrane. J. Biol. Chem. 279, 3627736286.

Morel, J., Claverol, S., Mongrand, S., Furt, F., Fromentin, J., Bessoule, J.-J., et al. (2006). Proteomics of plant detergent-resistant membranes. Mol. Cell. Proteomics 5, 1396 1411.

Ono, E., Wong, H. L., Kawasaki, T. Hasegawa, M., Kodama, O., and Shimamoto, K. (2001). Essential role of the small GTPase Rac in disease resistance of rice. Proc. Natl. Acad. Sci. U.S.A. 98, 759-764.

Ovecka, M., Berson, T., Beck, M., Derksen, J., Samaj, J., Baluska, F., etal. (2010). Structural sterols are involved in both the initiation and tip growth of root hairs in Arabidopsis thaliana. Plant Cell 22, 29993019.

Peskan, T., Westermann, M., and Oelmüller, R. (2000). Identification of low-density Triton X-100-insoluble plasma membrane microdomains in higher plants. Eur. J. Biochem. 267, 69896995.

Raffaele, S., Bayer, E., Lafarge, D., Cluzet, S., German Retana, S., Boubekeur, T., et al. (2009). Remorin, a solanaceae protein resident in membrane rafts and plasmodesmata, impairs potato virus $\mathrm{X}$ movement. Plant Cell 21, 15411555.

Schroeder, R., London, E., and Brown, D. (1994). Interactions between saturated acyl chains confer detergent resistance on lipids and glycosylphosphatidylinositol (GPI)anchored proteins: GPI-anchored proteins in liposomes and cells show similar behavior. Proc. Natl. Acad. Sci. U.S.A. 91, 1213012134.

Shahollari, B., Peskan-Berghofer, T. and Oelmuller, R. (2004). Receptor kinases with leucine-rich repeat are enriched in Triton X-100 insoluble plasma membrane microdomains from plants. Physiol. Plant. 122, 397-403.

Sharma, P., Sharma, N., and Deswal, R. (2005). The molecular biology of the low-temperature response in plants. Bioessays 27, 1048-1059.

Simons, K., and Ikonen, E. (1997). Functional rafts in cell membranes. Nature 387, 569-572.

Simons, K., and Toomre, D. (2000). Lipid rafts and signal transduction. Nat. Rev. Mol. Cell Biol. 1, 31-39.
Singer, S. J., and Nicolson, G. L. (1972). The fluid mosaic model of the structure of cell membranes. Science 175, 720-731.

Stanislas, T., Bouyssie, D., Rossignol, M., Vesa, S., Fromentin, J., Morel, J., etal. (2009). Quantitative proteomics reveals a dynamic association of proteins to detergent-resistant membranes upon elicitor signaling in tobacco. Mol. Cell. Proteomics 8, 2186-2198.

Takahashi, D., Kawamura, Y., Yamashita, T., and Uemura, M. (2012). Detergent-resistant plasma membrane proteome in oat and rye: similarities and dissimilarities between two monocotyledonous plants. J. Proteome Res. 11, 16541665.

Tanner, W., Malinsky, J., and Opekarová, M. (2011). In plant and animal cells, detergent-resistant membranes do not define functional membrane rafts. Plant Cell 23, 1191-1193.

Uemura, M., Tominaga, Y., Nakagawara, C., Shigematsu, S., Minami, A., and Kawamura, Y. (2006). Responses of the plasma membrane to low temperatures. Physiol. Plant. 126, 81-89.

Uemura, M., and Yoshida, S. (1984). Involvement of plasma membrane alterations in cold acclimation of winter rye seedlings (Secale cereale L. cv Puma). Plant Physiol. 75, 818-826.

Webb, M. S., Uemura, M., and Steponkus, P. L. (1994). A comparison of freezing injury in oat and rye: two cereals at the extremes of freezing tolerance. Plant Physiol. 104, 467-478.

Wong, H. L., Sakamoto, T., Kawasaki, T., Umemura, K., and Shimamoto, K. (2004). Down-regulation of metallothionein, a reactive oxygen scavenger, by the small GTPase OsRacl in rice. Plant Physiol. 135, 1447-1456.

Xu, X., Bittman, R., Duportail, G., Heissler, D., Vilcheze, C., and London, E. (2001). Effect of the structure of natural sterols and sphingolipids on the formation of ordered sphingolipid/sterol domains (rafts): comparison of cholesterol to plant, fungal, and disease-associated sterols and comparison of sphingomyelin, cerebrosides, and ceramide. J. Biol. Chem. 276, 33540-33546.

Yamazaki, T., Kawamura, Y., Minami, A., and Uemura, M. (2008). Calcium-dependent freezing tolerance in Arabidopsis involves membrane resealing via synaptotagmin SYT1. Plant Cell 20, 3389-3404

Conflict of Interest Statement: The authors declare that the research was 
conducted in the absence of any commercial or financial relationships that could be construed as a potential conflict of interest.

Received: 28 December 2012; paper pending published: 15 January 2013; accepted:
05 February 2013; published online: 22 February 2013.

Citation: Takahashi D, Kawamura Y and Uemura M (2013) Detergent-resistant plasma membrane proteomes to elucidate microdomain functions in plant cells.
Front. Plant Sci. 4:27. doi: 10.3389/fpls. 2013.00027

This article was submitted to Frontiers in Plant Proteomics, a specialty of Frontier in Plant Science.

Copyright (C) 2013 Takahashi, Kawamura and Uemura. This is an open-access article distributed under the terms of the Creative Commons Attribution License, which permits use, distribution and reproduction in other forums, provided the original authors and source are credited and subject to any copyright notices concerning any third-party graphics etc. 